\title{
Large-Scale Sublattice Asymmetry in Pure and Boron-Doped Graphene
}

Dmitry Yu. Usachov, ${ }^{\dagger}$ Alexander V. Fedorov, ${ }^{\dagger, \pm}, \pi$ Oleg Yu. Vilkov, ${ }^{\dagger}$ Anatoly E. Petukhov, ${ }^{\dagger}$ Artem G. Rybkin, ${ }^{\dagger}$ Arthur Ernst, ${ }^{\S}$ Mikhail M. Otrokov, ${ }^{\|,{ }_{\perp}{ }^{\dagger}}$ Evgueni V. Chulkov, ${ }^{\dagger}, \|_{\perp}{ }_{\perp}$ lya I. Ogorodnikov, ${ }^{\#}$ Mikhail V. Kuznetsov, ${ }^{\#}$ Lada V. Yashina,${ }^{@}$ Elmar Yu. Kataev, ${ }^{@}$ Anna V. Erofeevskaya, ${ }^{\dagger}$ Vladimir Yu. Voroshnin, ${ }^{\dagger}$ Vera K. Adamchuk, ${ }^{\dagger}{\text { Clemens Laubschat, }{ }^{\Delta} \text { and Denis V. Vyalikh }{ }^{\dagger} \|, \Delta, \nabla}$

† St. Petersburg State University, 7/9 Universitetskaya nab, St. Petersburg, 199034, Russia ‡ Physikalisches Institut, Universität zu Köln, Zülpicher Strasse 77, 50937 Köln, Germany II IFW Dresden, P.O. Box 270116, D-01171 Dresden, Germany

§ Max-Planck-Institut für Mikrostrukturphysik, Weinberg 2, D-06120 Halle, Germany // Donostia International Physics Center (DIPC), Departamento de Fisica de Materiales and CFM-MPC UPV/EHU, 20080 San Sebastian, Spain

$\perp$ Tomsk State University, Lenina Av., 36, 634050 Tomsk, Russia \# Institute of Solid State Chemistry of the Ural Branch of the Russian Academy of Sciences, Pervomayskaya Str. 91, 620990 Ekaterinburg, Russia @ M.V. Lomonosov Moscow State University, Leniskie Gory 1/3 199991 Moscow, Russia $\Delta$ Institute of Solid State Physics, Dresden University of Technology, 01062 Dresden, Germany $\nabla$ IKERBASQUE, Basque Foundation for Science, 48011 Bilbao, Spain

\section{Supporting Information}



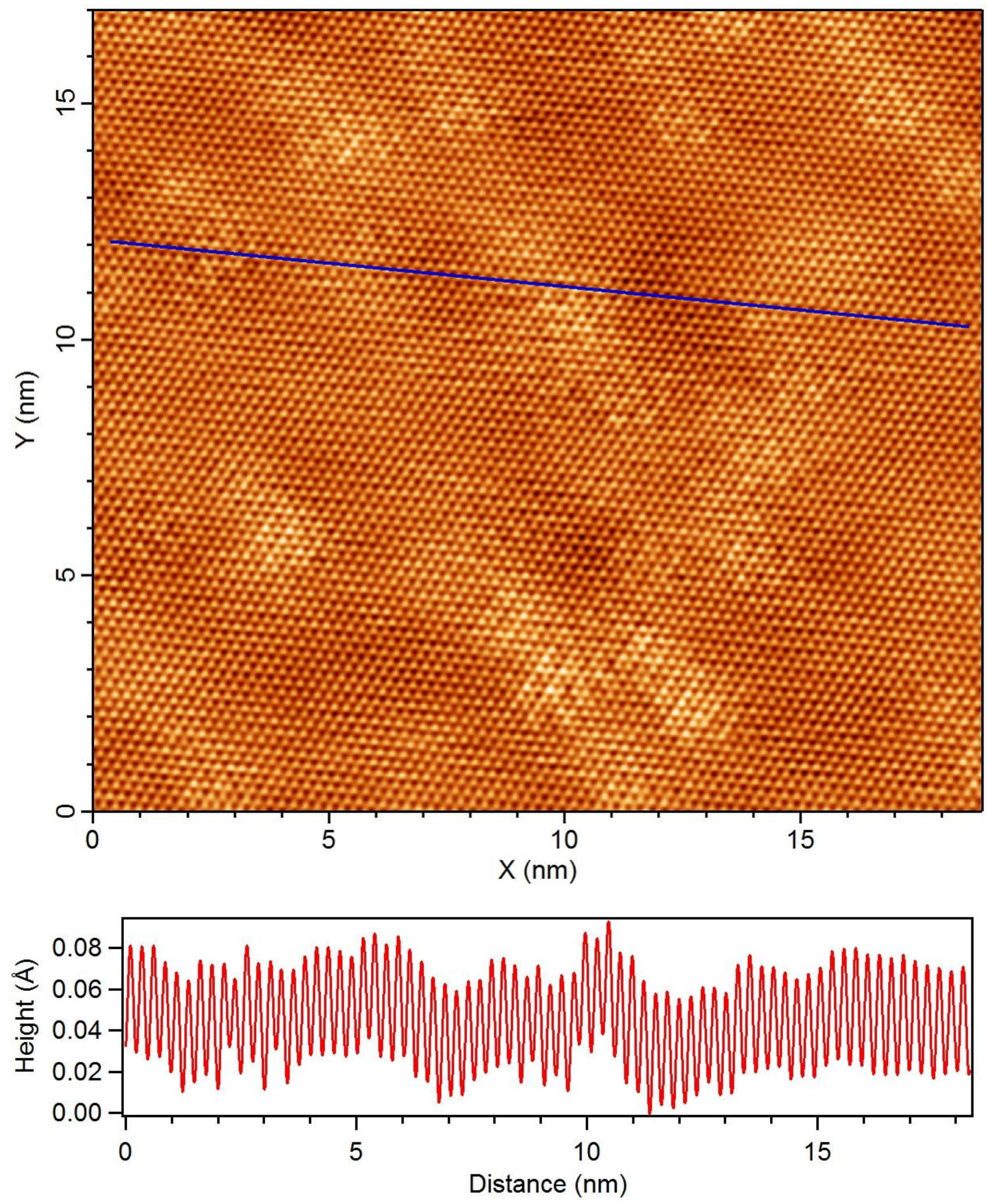

Figure S1. Atomically-resolved STM image of the graphene/Co(0001) system, demonstrating sublattice asymmetry. It can be seen that the local scale of sublattice asymmetry exceeds the size of the image $(\sim 20 \mathrm{~nm})$. The profile of the STM image, marked with a blue line, shows negligible corrugations of graphene on the scale of few nanometers. 